\title{
US Spanish
}

National Cancer Institute

\section{Source}

National Cancer Institute. US Spanish. NCI Thesaurus. Code C160189.

A form of the Spanish language spoken in the United States. 